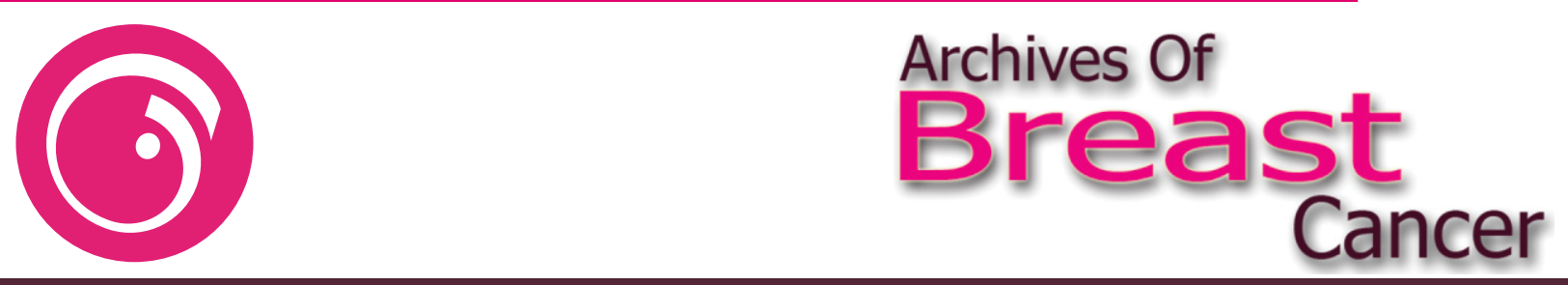

DOI: $10.32768 / a b c .20196151-54$

Comparing BI-RADS 2013 With the 2003 Edition: Has It Worked Better?

\author{
Afsaneh Alikhassi ${ }^{* a}$ \\ a Department of Radiology, Cancer Institute, Imam Khomeini Hospital, Tehran University of Medical Sciences (TUMS), \\ Tehran, Iran
}

The most recent (5th) edition of the American College of Radiology (ACR) Breast Imaging Reporting and Data System (BI-RADS) Atlas has replaced the previous one in most centers. ${ }^{1}$ Various texts and articles in the literature have reviewed or summarized the new edition, and some original articles have evaluated the predictive value of BIRADS descriptors. In this commentary, we highlight some problems that we had with the new edition of BI-RADS in daily clinical practice.

We believe the changes introduced into the latest edition of the ACR Atlas can be classified into minor changes, completing changes, and major challenging changes. ${ }^{2}$

\section{Minor Changes}

Minor changes can be further categorized into three groups: (1) lexical changes, such as using rim calcification instead of eggshell and lucent-centered calcification, asymmetry instead of simple asymmetry, which is mostly to make reporting easier; (2) changes in classifications, i.e., moving global asymmetry from the "special cases" category to the "asymmetries" category or taking intermammary lymph nodes out of the "special cases" category and making it an independent category; and (3) addition of new items such as simple cyst and fat necrosis to the "special cases" category. These changes are easily substituted.

\section{Adding and Completing Data}

Some new useful parts were added to the latest edition of BI-RADS, including a background on parenchymal enhancement in MRI (minimal, mild,

\footnotetext{
* Address for correspondence:

Afsane Alikhassi, MD

Assistant professor,

Address: Department of Radiology, Cancer Institute, Imam

Khomeini Hospital, Keshavarz Blvd., Tehran, 3314114197, Iran

Tel: +98 2161192849

Fax: +982166581626

Email: afsanehalikhassi@yahoo.co.uk
}

moderate, or marked) and a new subsection on prosthesis assessment. The previously deficient parts of the BI-RADS atlas have now been completed.

\section{Major Changes}

Major changes are those that had a great impact on daily clinical practice and are as follows: (1) density description; (2) omitting the "intermediate concern" group from calcifications categories, and BI-RADS 4a; and (3) BI-RADS 3, 4, and 5 should only be used after a full imaging workup and not for mammography screening.

\section{Density}

The 2003 edition of BI-RADS used breast composition categories (ACR category 1-4) based on the overall density ( $\%$ ) of fibrograndular tissue as a breast cancer risk indicator. However, the ACR Committee on BI-RADS concluded that the association of breast density with the sensitivity of mammography is clinically more important than the percentage of breast density as an indicator for breast cancer risk (Figure 1). Therefore, in the 2013 edition, new breast composition categories (assigned alphabetically $a-d$ to avoid possible confusion with the numbered BI-RADS assessment categories), have been introduced.

There is intra- and inter-observer variation in visually estimating breast density between any two adjacent density categories in both types of categorization. In a study performed in our center, substantial inter-observer agreement was seen using both the fourth and fifth editions, and the intraobserver agreement was high for both editions. The percentage of women who were classified as having dense breasts was also not statistically significant using both methods. ${ }^{3}$

\section{$B I-R A D S 3$}

Since the release of the ACR BI-RADS fifth 


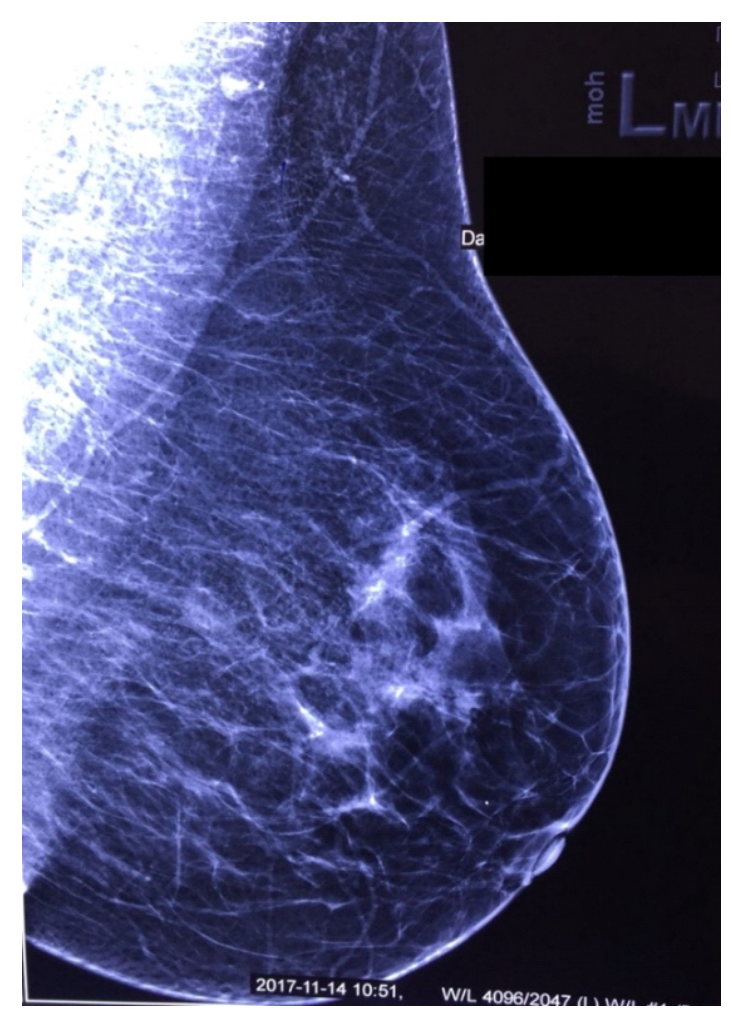

Figure 1. This left breast mediolateral mammography has $25 \%$ to $50 \%$ fibroglandular tissue (ACR 2 based on the BI-RADS 2003 edition), and it has a small amount of density that can obscure a small lesion, which would result in breast composition category $\mathrm{C}$ according to the current edition.

edition, when a patient attends our center for screening or a benign-appearing lesion, such as when an oval circumscribed mass is found, we classify it as BI-RADS 0 in the report and perform a full evaluation including targeted sonography. The authors of the BI-RADS 2013 believed that some of these patients would not need short-term follow-up mammography after a full evaluation. For example, the mass may be identified as a cyst on the targeted sonogram (Figure 2). On rare occasions, the described mass can be a solid circumscribed one on sonography with a high flow in a simultaneous color Doppler, which suggests a high-grade malignancy. Some of these lesions may have a solid oval circumference and appear to have a homogenous texture on sonography; these will obtain a classification of BI-RADS 3. In this case, the patient will be recommended to have a short-term follow-up via sonography. In palpable lesions with a BI-RADS 3 appearance on imaging, different clinicians have different approaches because of nonrobust evidence in the literature, with some preferring to order a biopsy. As mentioned in the BI-RADS edition 5, and based on our experience, persistent focal asymmetry after full evaluation and a group of punctate microcalcifications are the most prevalent mammographic BI-RADS 3 classifications that

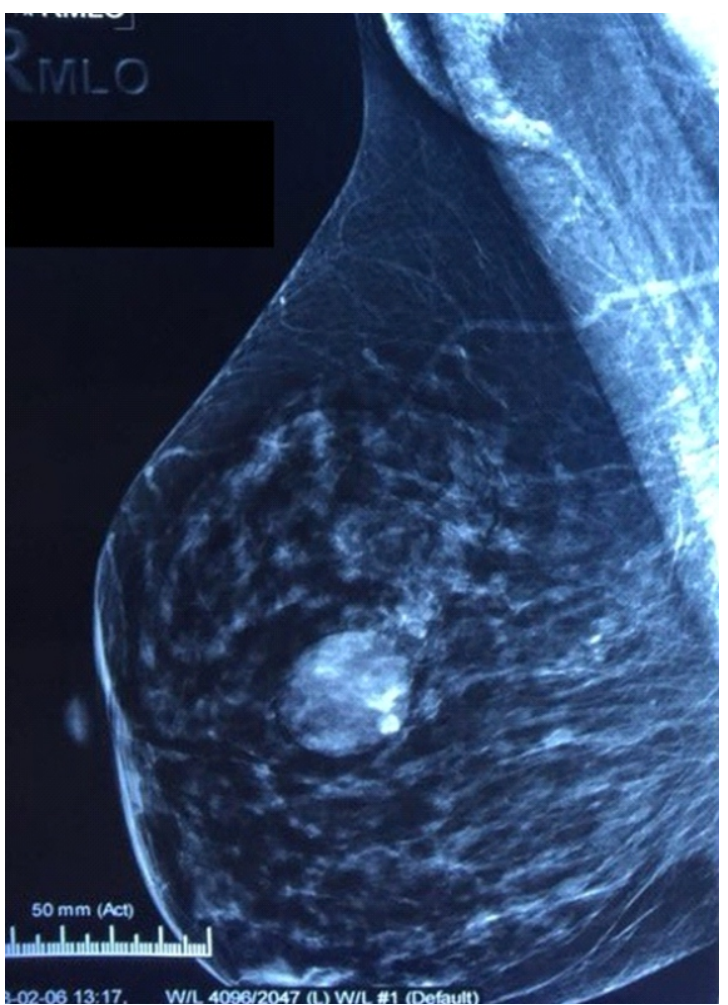

Figure 2. Mediolateral oblique mammography of the right breast shows an oval-shaped mass in central part of the right breast. The mass was identified as a cyst on targeted sonography, with no need for further short-term follow-up.

need short-term follow-up with mammography.

\section{$B I-R A D S 4$ and 5}

In the previous BI-RADS edition, categories 4 and 5 could not be reported during a screening mammography. Both categories required additional images before they could be classified. However, in some imaging departments, such as our center in Iran, the screening and diagnostic wards are not separate, and the same radiologists often perform both tasks on the same day. In addition, for insurance and legal reasons, the radiologists are not supposed to take any additional images without the request of a clinician. Thus, if we encounter a suspicious or highly suspicious finding, we will assign it to BI-RADS 4 or 5 category based on the descriptors in the screening mammography. For example, if we detect a spiculated mass containing suspicious microcalcifications during a screening mammography, we assign a classification of BI-RADS 5 instead of BI-RADS 0 in the same session (Figure 3).

\section{Calcifications}

We believe that the most challenging changes in the BI-RADS 2013 as compared to the 2003 edition are reporting microcalcifications. The changes are as 


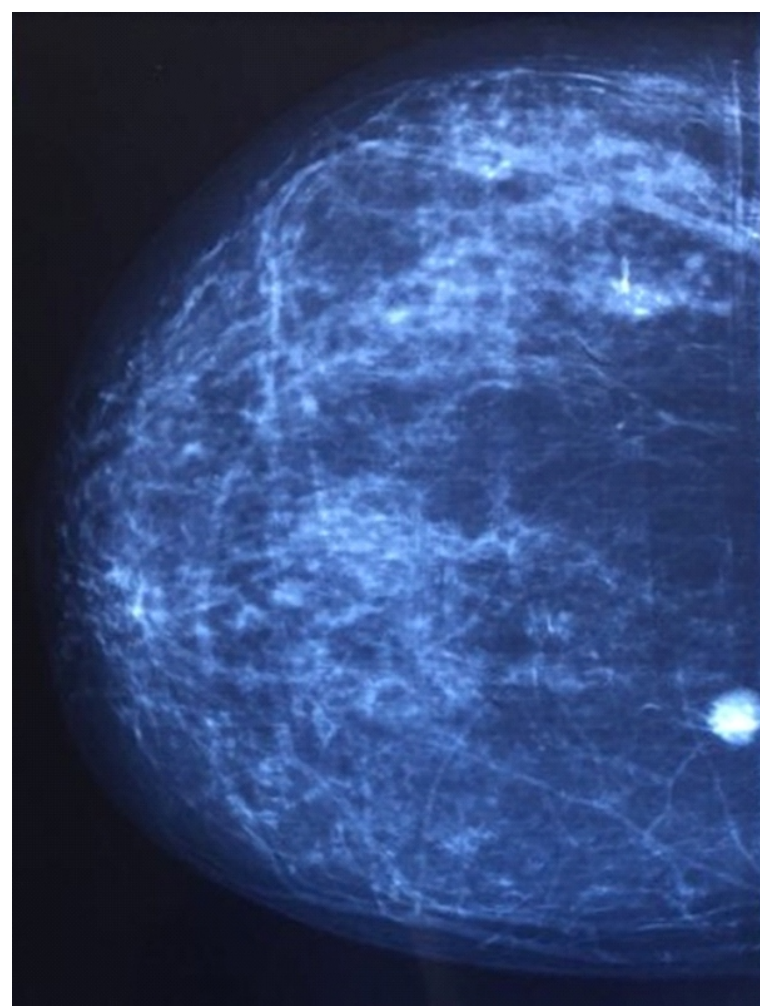

Figure 3. Craniocaudal view of a 48-year-old woman's screening mammography of the right breast shows a dense spiculated mass in the deep inner part, and a classification of BI-RADS 5 was assigned.

follows:

- In the 2013 edition, "lucent-centered" and "eggshell" calcifications in the "typically benign" category have been replaced by the new term "rim" for ease and simplicity.

- The 2013 edition changed the upper size limit for the microcalcification group definition to $2 \mathrm{~cm}$ while retaining $>2 \mathrm{~cm}$ as the lower limit for regional distribution to eliminate a previous gap. Additionally, the 2013 edition describes size measurement as a linear dimension instead of a volume.

- Solitary group of punctate microcalcifications, which is probably a benign assessment with a shortterm follow-up requirement, has been placed in BIRADS 3 category. If a similar group of calcifications is new, increasing, or adjacent to a known cancer, it warrants a biopsy.

- One major change in microcalcification classifications in the 2013 edition is the omitting of the "intermediate concern" category. The three calcification types (amorphous, coarse heterogeneous, and fine pleomorphic) are grouped as "suspicious morphology," and since their probability of malignancy is $10 \%$ to $50 \%$, they are assigned BI-RADS $4 \mathrm{~b}$. Fine linear or fine linear branching calcifications are placed in category $4 \mathrm{c}$ or 5 depending on their distribution.

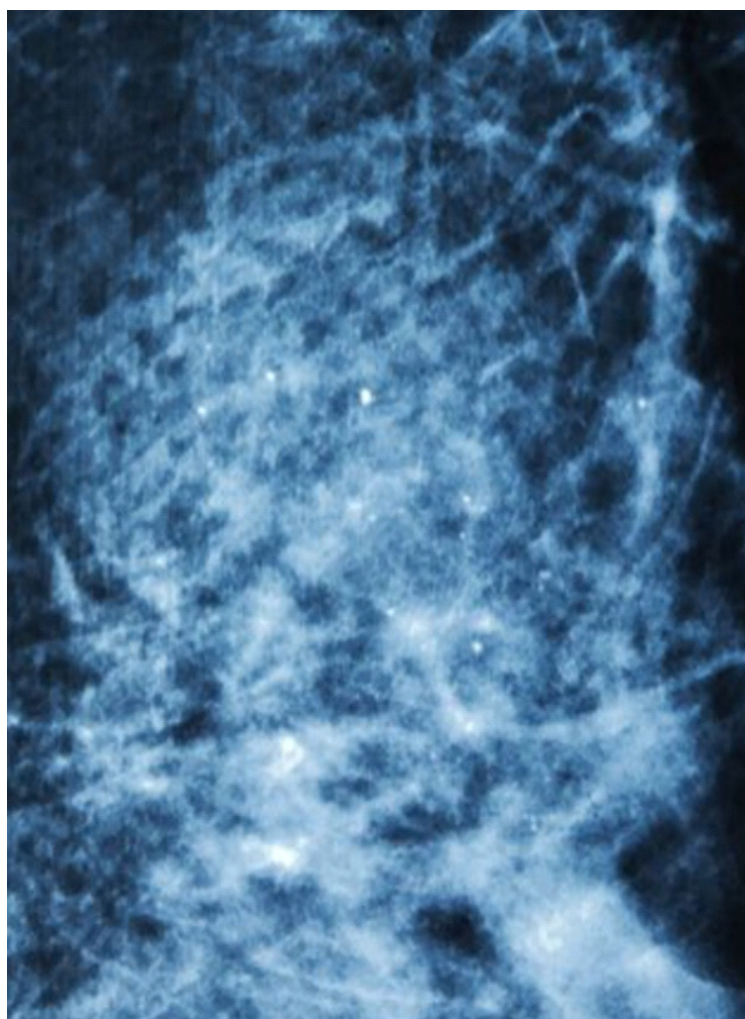

Figure 4. A focal compression view showing a regional group of punctate microcalcifications with some heterogeneity in size and density, and it is not obvious which BI-RADS classification should be assigned to this lesion.

However, problems still remain in the ACR

BI-RADS 2013 regarding the reporting of microcalcifications:

- The number of microcalcifications in each group is not identified, but we know that when their number increases, the probability of malignancy will increase.

- Although some types of microcalcifications encountered in daily clinical practice are not mentioned, such as punctate microcalcifications with regional distribution and regionally distributed microcalcifications with some degree of pleomorphism. This may be because there is little robust data in the literature.

- Heterogeneity in the density and size of microcalcifications were also not focused. For example, it is not clear which BI-RADS score should be assigned to a group of punctate microcalcifications varying in size and density (Figure 4).

In summary, the BI-RADS system has greatly improved breast imaging reporting by standardizing the reports and has made data collection for research purposes much easier, but some other important details may be considered in the future editions.

\section{Conflict of Interest}

None. 


\section{References}

1. D'Orsi CJ, Sickles EA, Mendelson EB, Morris EA, al. e. ACR BI-RADS ${ }^{\circledR}$ Atlas, Breast Imaging Reporting and Data System. Reston, VA, American College of Radiology 2013.

2. ACR BI-RADS Atlas 5 Edition Changes.Available at:[https://www.acr.org/ Clinical-Resources/Reporting-and-DataSystems/Bi-Rads]

3. Alikhassi A, Gourabi HE, Baikpour M. Comparison of inter-and intra-observer variability of breast density assessments using the fourth and fifth editions of Breast Imaging Reporting and Data System. European journal of radiology open. 2018;5:67-72.

4. Burnside ES, Ochsner JE, Fowler KJ, Fine JP, Salkowski LR, et al. Use of microcalcification descriptors in BI-RADS 4th edition to stratify risk of malignancy. Radiology. 2007;242(2): 388-95.

5. Bent CK, Bassett LW, D'Orsi CJ, Sayre JW. The positive predictive value of BI-RADS microcalcification descriptors and final assessment categories. American Journal of Roentgenology. 2010;194(5):1378-83.

6. Liberman L, Abramson AF, Squires FB, Glassman $\mathrm{J}$, Morris E, et al. The breast imaging reporting and data system: positive predictive value of mammographic features and final assessment categories. AJR American journal of roentgenology. 1998;171(1):35-40.

7. Berg WA, Arnoldus CL, Teferra E, Bhargavan M. Biopsy of amorphous breast calcifications: pathologic outcome and yield at stereotactic biopsy. Radiology. 2001;221(2):495-503.

8. Ahmadinejad N, Jafari M, Rahmani M, RezaeiKalantari K, Shakiba M. Evaluation of Suspicious Appearing Microcalcification Groups on Mammogram: Comparing BIRADS 5th Edition Descriptors with the BIRADS 4th Edition and Pathologic Association. Lateral. 2018;5:5.7.

9. Egan R, McSweeney M, Sewell C.Intramammary calcifications without an associated mass inbenign and malignant diseases. Radiology. 1980;137(1):1-7. 